\title{
AN OPTIMAL CONTROL PROBLEM IN ECONOMICS
}

\author{
JANNETT HIGHFILL \\ Department of Economics \\ Bradley University \\ Peoria, Illinois 61625 \\ and \\ Michael Mcasey \\ Department of Mathematics \\ Bradley University \\ Peoria, Illinois 61625 \\ (Received February 5, 1990 and in revised form July 2, 1990)
}

\begin{abstract}
The first problem in the economics of natural resources is to find the rate at which to extract the resource in order to optimize its value when there are no extraction costs. It is shown that the existence of an optimal extraction path is not guaranteed by a utility function that is merely (strictly) concave, but that the additional requirement of "asymptotic nonlinearity" will assure the existence of the desired optimum.
\end{abstract}

KEY WORDS AND PHRASES. Existence, optimal control, nonrenewable resource. 1980 AMS (MOS) SUBJECT CLASSIFICATION: 49A10, 90A16

\section{INTRODUCTION.}

The first problem in the economics of exhaustible natural resources is to establish the extraction rate of the resource. This problem is formulated in the language of the calculus of variations or, more commonly, as an optimal control problem: choose an extraction rate $q(\mathrm{t})$ to maximize the total discounted utility of the resource, $\int_{0}^{\infty} \mathrm{U}(\mathrm{q}(\mathrm{t})) \mathrm{e}^{-\delta \mathrm{t}} \mathrm{dt}$, subject to appropriate initial conditions. The subject of this paper is the existence of a solution to this problem. Existence theorems for optimal control problems are abundant in the literature, but the simplicity of the problem considered here demands a simple answer.

It is well known that even in the simple setting described above, the existence of an 
optimal solution is not always assured. Indeed, if the utility function is linear, a solution does not exist that is "usable" for a resource extractor. Although concavity is the natural hypothesis for the utility function $U$, the existence of an optimal extraction path is not guaranteed by the strict concavity of $\mathrm{U}$. It will be shown that the additional geometric requirement of "asymptotic nonlinearity" will resolve these two difficulties and assure the existence of the desired optimum.

Optimal extraction problems have been discussed extensively in the literature. Although more sophisticated models have been developed, the one here is always the first discussed; see, for example, the text by Dasgupta and Heal [1] and the references therein. Two recent works that include a discussion of existence for this simple resource extraction problem are Epstein's 1983 paper [2] and Lozada's 1987 thesis [3]. Both these works consider the optimal control problem from the point of view of an unbounded horizon problem and so are more general than the paper of Yaari [4] on the finite horizon problem. More importantly, as both Epstein and Lozada realize, the key to existence is exhaustion. The example at the end of section 2 will illustrate this. The condition of asymptotic nonlinearity introduced here is a simple geometric condition on the utility function, $U(q)$, saying essentially that $U(q)$ does not become too linear for $q$ large. This is sufficient to guarantee exhaustion and hence, using a result of Toman [5], existence is assured. It is easy to show that this condition is in fact equivalent to Epstein's two integral conditions in his Lemma 1, but the condition here is more easily verified and has a simple geometric interpretation. The condition of asymptotic nonlinearity is distinct from Lozada's conditions as examples will show. Finally recent results of Botteron and Dacorogna [6] in the theory of optimal foraging provide conditions similar in spirit to ours. Their results are complementary to ours in that their problem has a fixed (finite) terminal time and a fixed terminal state.

In section 2, notation and preliminary results will be established. A brief proof of a "folk theorem" is given which provides a simple criterion to determine if extraction occurs on a finite or infinite interval. An example is given at the end of section 2 of a strictly concave utility function for which the associated optimal control problem does not have a solution. In the second section it is shown that if the utility function is strictly concave and "asymptotically nonlinear" then an optimal solution exists. The theorem is compared with the results of Epstein [2], Lozada [3], and Botteron and Dacorogna [6].

\section{STATEMENT OF THE PROBLEM AND PRELIMINARY RESULTS.}

A utility function is a map $\mathrm{U}: \mathbb{R}_{+} \rightarrow \mathbb{R}$ satisfying the following conditions:

i) $U$ is twice continuously differentiable;

ii) $U \geq 0$;

iii) $\mathrm{U}^{\prime}>0$; and

iv) $\mathrm{U}^{\prime \prime}<0$.

The objective is to maximize the present value of the utility of an exhaustible resource. Let $\mathrm{x}(\mathrm{t})$ be the amount of the resource remaining in situ at time $t$. The initial amount of the resource is $x_{0}$. Let $q(t)$ denote the extraction rate. The problem is to choose $q$ (and implicitly an extraction time $\mathrm{T} \leq \infty$ ) in order to maximize: 


$$
\int_{0}^{T} \mathrm{U}(\mathrm{q}(\mathrm{t})) \mathrm{e}^{-\delta \mathrm{t}} \mathrm{dt} \text { subject to the conditions } \frac{d \mathrm{x}}{\mathrm{dt}}=-\mathrm{q}, \mathrm{x}(0)=\mathrm{x}_{\mathrm{o}}, \mathrm{x}(\mathrm{T}) \geq 0, \mathrm{q} \geq 0 .
$$

Since $U$ is increasing and nonnegative, if an optimal path exists, the resource will always be exhausted: $x(T)=0$. This fact will imply the non-existence of an optimal extraction path for the utility function constructed at the end of this section. The following proposition summarizes some well known necessary conditions for optimal extraction paths.

PROPOSITION 2.1. A) If $x(t)$ is optimal, then $\lim _{t \rightarrow T^{-}} x(t)=0$.

B) Let $q$ be an optimal extraction rate. Then

i) $\mathrm{q}(\mathrm{t})>0$, for $0<\mathrm{t}<\mathrm{T} ; \mathrm{q}(\mathrm{t})=0$ for $\mathrm{t}>\mathrm{T}$;

ii) $q(t)$ is a continuous, decreasing function on $[0, T]$;

iii) $\mathrm{q}(\mathrm{T})=0$.

Finally, it is important in problems of resource extraction to know if the time to exhaustion is finite or infinite. This is especially critical since the transversality conditions often differ in the finite and infinite cases. In the following theorem, it is shown that the value of the derivative of the utility function at $q=0$ provides a simple test to determine the extent of the extraction horizon.

THEOREM 2.2. Assume an optimal solution exists. The extraction horizon is finite if and only if the derivative $U^{\prime}$ is bounded (at $q=0$ ).

PROOF. The Hamiltonian for the problem is $H=U(q) e^{-\delta t}-\lambda q$. The Maximum Principle guarantees the existence of the constant $\lambda$. Apply the Mean Value Theorem to $H$ as follows. Let $\mathrm{q}$ be a feasible extraction function (nonnegative and piecewise continuous). Fix $t$. Then

$$
\begin{aligned}
\mathrm{H}(0)-\mathrm{H}(\mathrm{q}(\mathrm{t})) & =[\mathrm{U}(0)-\mathrm{U}(\mathrm{q}(\mathrm{t}))] \mathrm{e}^{-\delta t}+\lambda \mathrm{q}(\mathrm{t}) \\
& =\mathrm{U}^{\prime}(\tilde{\mathrm{q}})(-\mathrm{q}(\mathrm{t})) \mathrm{e}^{-\delta \mathrm{t}}+\lambda \mathrm{q}(\mathrm{t}), \text { by the Mean Value Theorem } \\
& \text { where } 0<\tilde{\mathrm{q}}<\mathrm{q}(\mathrm{t}) \\
& =-\mathrm{q}(\mathrm{t})\left[\mathrm{U}^{\prime}(\tilde{\mathrm{q}}) \mathrm{e}^{-\delta \mathrm{t}}-\lambda\right]
\end{aligned}
$$

If $\mathrm{U}^{\prime}(0)=+\infty$ (i.e. $\lim _{\mathrm{q} \rightarrow 0^{+}} \mathrm{U}^{\prime}(\mathrm{q})=+\infty$ ), we want to show that it is not optimal to have $q(T)=0$ for any finite $T$. Fix $T$ and construct a feasible path $q$ with $q(T)>0$ but so small that $\lambda \mathrm{e}^{\delta \mathrm{t}}<\mathrm{U}^{\prime}(\mathrm{q}(\mathrm{T}))$. Then $\mathrm{H}(0)-\mathrm{H}(\mathrm{q}(\mathrm{T}))<0$, so by the Maximum Principle, the optimal solution cannot be zero at $\mathrm{T}$.

On the other hand, if $\mathrm{U}^{\prime}(0)<\infty$, we want to show that the optimal solution will be zero for large $t$. Since $U^{\prime}(0)$ is bounded, for any extraction function $q$, the value of $t$ can be chosen so large that $U^{\prime}(q(t)) e^{-\delta t}-\lambda<0$. Thus for $q(t)>0, H(0)-H(q(t))>0$ and so $q(t)>0$ is not optimal.

The final item of this section is an example showing that the Maximum Principle fails to yield the optimum extraction path for a class of utility functions satisfying assumptions (i)-(iv). A linear function is a trivial example of a utility function for which the Maximum 
Principle yields no information, although such a function also fails to satisfy conditions (iii) and (iv). With a linear utility function, the present value of utility is always increased by extracting the same quantity of resource over a shorter time. (So the "optimal" extraction decision would be to extract all of the resource at the first instant. Although point masses are well known to arise as solutions to optimal control problems, they are not solutions that can be implemented by a resource extractor.) The problem with a linear utility function would disappear if the feasible extraction rates were uniformly bounded. However without introducing other considerations into the model (eg. costs), there are no a priori upper bounds on the extraction rates which are justified from the economics of the problem. This issue can also arise for utility functions which are strictly concave, as shown in the following example. The example is similar to one by Yaari [1964].

EXAMPLE 2.3. Let $\mathrm{U}(\mathrm{q})=-\mathrm{e}^{-\mathrm{q}}+\mathrm{q}+1$. The Hamiltonian is

$$
\mathrm{H}=\mathrm{e}^{-\delta \mathrm{t}}\left[-\mathrm{e}^{-\mathrm{q}}+\mathrm{q}+1\right]-\lambda \mathrm{q} .
$$

Applying the Maximum Principle yields $\lambda=\mathrm{e}^{-\delta \mathrm{t}}\left(\mathrm{e}^{-\mathrm{q}}+1\right)$. Solving this for $\mathrm{q}$ gives $q=-\ln \left(\lambda \mathrm{e}^{-\delta \mathrm{t}}-1\right)$. Since $\mathrm{q}(\mathrm{T})=0$ if an optimal solution exists, it follows that

$$
\lambda=2 \mathrm{e}^{-\delta \mathrm{T}} \quad \text { and } \mathrm{q}(\mathrm{t})=-\ln \left(2 \mathrm{e}^{-\delta(\mathrm{T}-\mathrm{t})}-1\right) .
$$

The issue here is the existence of an upper bound for $q$. From the form of $q$ it is seen that the existence of an optimal extraction rate depends on the terminal time $T$. Such a time $T$ is found by solving, if possible, the following equation representing exhaustion of the resource:

$$
x_{0}=\int_{0}^{T} q(t) d t=\int_{0}^{T}-\ln \left(2 e^{-\delta(T-t)}-1\right) d t .
$$

Since $q$ must be nonnegative, there is an upper bound on $T$, namely $T \leq T_{m} \equiv \ln (2) / \delta$. Thus equation $(2.1)$ can be solved for all stock sizes, $x_{0}$, less than some maximum, $x_{m}$. Since $T_{m}$ is known, $x_{m}$ can be computed explicitly in this example by a change of variables:

$$
x_{m}=\int_{0}^{T_{m}}-\ln \left(2 e^{-\delta\left(T_{m}-t\right)}-1\right) d t=\frac{\pi^{2}}{12, \delta} .
$$

So for small stock sizes $\left(x_{0} \leq x_{m}\right)$, the optimal extraction path is determined by the first order conditions. Moreover, the value of the resource is given by

$$
\begin{aligned}
\mathrm{V}\left(\mathrm{x}_{\mathrm{o}}\right) & =\int_{0}^{\mathrm{T}}\left(-\mathrm{e}^{-\mathrm{q}}+\mathrm{q}+1\right) \mathrm{e}^{-\delta \mathrm{t}} \mathrm{dt}=\int_{0}^{\mathrm{T}}-\ln \left(2 \mathrm{e}^{-\delta(\mathrm{T}-\mathrm{t})}-1\right) \mathrm{e}^{-\delta \mathrm{t}} \mathrm{dt} \\
& =\delta^{-1}\left[1-\mathrm{e}^{-\mathrm{q}(0)}-\mathrm{q}(0) \mathrm{e}^{-\mathrm{q}(0)}\right] .
\end{aligned}
$$

However for $x_{0}>x_{m}$, a solution $T$ does not exist for equation (2.1). Thus for stock sizes greater than $x_{m}$, no solution will exhaust the resource, and so by Proposition 2.1, no optimal solution exists.

Finally note that in case $x_{0}=x_{m}$, the stock constraint $(2.1)$ is exactly satisfied and the first order condition implies that $q$ is always nonnegative. However equation (2.1) 
implies that the initial rate of extraction is $q(0)=+\infty$. Further, although an upper bound exists for the value function $\mathrm{V}\left(\mathrm{x}_{0}\right)$ (in fact $\mathrm{V}\left(\mathrm{x}_{\mathrm{o}}\right) \leq 1 / \delta$ ) that limit is not reached if $q(0)$ is finite. Since extraction rates are, by definition, finite everywhere, no optimal solution exists in case $x_{0}=x_{m}$.

\section{EXISTENCE OF OPTIMAL EXTRACTION PATHS.}

It is routine to check that if the admissible extraction functions $q(t)$ are required to take on values in a bounded set (e.g. $0 \leq q(t) \leq B$ ) then, with the usual assumptions on $U$, there exists an optimal path. (See Theorem 2 of Toman [5].) The preceding example shows that the boundedness requirement is nontrivial. Moreover, in the absence of extraction costs, there are no a priori bounds on the extraction rate. However, as suggested by Toman $[5, \S 7]$, it may be possible for bounds to be inferred from other aspects of the problem. This is done next. The goal is to show that the resource will be exhausted. This will imply that the initial extraction rate is finite and hence $q(t)$ is bounded since the extraction function is monotone. One additional assumption is needed on the utility function $U$. The assumption is basically that the asymptotic behavior of $U$ is not linear. This assumption is used to guarantee that the resource will be exhausted (i.e. $\int \mathrm{q}(\mathrm{t}) \mathrm{dt}=\mathrm{x}_{\mathrm{o}}$.) This in turn provides the required boundary conditions needed to solve the optimal control problem.

In order to state the main theorem, two pieces of notation are needed. Let $\beta$ $=\lim _{\mathrm{q} \rightarrow 0^{+}} \mathrm{U}^{\prime}(\mathrm{q})$, if this limit exists; otherwise let $\beta=+\infty$. Similarly let $\alpha=\lim _{\mathrm{q} \rightarrow \infty} \mathrm{U}^{\prime}(\mathrm{q})$. Since $U^{\prime}$ is monotone, this limit always exists. Abusing notation slightly, write $U^{\prime}(0)=$ $\beta$ and $\mathrm{U}^{\prime}(\infty)=\alpha$. The condition that $\mathrm{U}$ be asymptotically nonlinear is simply that $\lim _{\mathrm{q} \rightarrow \infty} \mathrm{U}(\mathrm{q})-\alpha \mathrm{q}=+\infty$. This means that the utility function is "not too linear" for large values of $q$.

THEOREM 3.1. Assume $U$ satisfies conditions (i) - (iv). Let $q(t)$ satisfy the first order conditions from the Maximum Principle. In case $\alpha>0$, assume additionally that $\mathrm{U}$ satisfies $\lim _{\mathrm{q} \rightarrow \infty} \mathrm{U}(\mathrm{q})-\alpha \mathrm{q}=+\infty$. Then the stock will be exhausted. Furthermore, $\mathrm{q}(0)<\infty$.

PROOF. Since $q(t)$ satisfies the first order conditions, the Hamiltonian $H=U(q) e^{-\delta t}$ - $\lambda \mathrm{q}$ is maximized as a function of $\mathrm{q}$. Therefore $\mathrm{q}(\mathrm{t})=\mathrm{W}\left(\lambda \mathrm{e}^{\delta \mathrm{t}}\right)$ where $\mathrm{W}=\left(\mathrm{U}^{\prime}\right)^{-1}$. There are two constants left to be determined: $\lambda$ and $T$. The terminal condition on $q$ $\left(\lim _{t \rightarrow T} q(t)=0\right)$, from the first order conditions allows for computation of $T$ as a function of

$\lambda$. (It is convenient for the proof to find $\mathrm{T}$ and $\lambda$ in this order.) Finally, the domain of $\mathrm{W}$ is the interval $(\alpha, \beta)$ so that $\alpha \leq \lambda \mathrm{e}^{\delta \mathrm{t}} \leq \beta$, for all $\mathrm{t} \in[0, \mathrm{~T}]$ and so $\lambda \in[\alpha, \beta]$. To determine $\lambda$, use the stock constraint: $x_{0}=\int_{0}^{T} q(t) d t$.

The problem of existence then reduces to the solution of the stock constraint for the multiplier $\lambda$ : 


$$
x_{0}=\int_{0}^{T} W\left(\lambda e^{\delta t}\right) d t
$$

To show that (3.1) has a solution $\lambda$, observe first that the integral $\int_{0}^{T} W\left(\lambda e^{\delta t}\right) d t$ is a continuous function of $\lambda$. To show that the stock can be exhausted, we show that

i) $\lim _{\lambda \rightarrow \alpha} \int_{0}^{T} W\left(\lambda e^{\delta t}\right) d t=+\infty$ and

ii) $\lim _{\lambda \rightarrow \beta} \int_{0}^{T} \mathrm{~W}\left(\lambda \mathrm{e}^{\delta \mathrm{t}}\right) \mathrm{dt}=0$.

To verify i) and ii), it is helpful to change variables: $v=\lambda e^{\delta t}$ so that

$$
\int_{0}^{T} W\left(\lambda e^{\delta t}\right) d t=\int_{\lambda}^{\beta} \frac{W(v)}{v} d v
$$

Assertion i. $\lim _{\lambda \rightarrow \alpha^{+}} \int_{\lambda}^{\beta} \frac{\mathrm{W}(\mathrm{v})}{\mathrm{v}} \mathrm{dv}=+\infty$.

Since $\beta$ may be infinite, it will be convenient to fix $\gamma \in(\alpha, \beta)$. The assertion is easily verified if $\alpha=0$. In this case

$$
\int_{\lambda}^{\beta} \frac{\mathrm{W}(\mathrm{v})}{\mathrm{v}} \mathrm{dv} \geq \int_{\lambda}^{\gamma} \frac{\mathrm{W}(\mathrm{v})}{\mathrm{v}} \mathrm{dv} \geq \mathrm{W}(\gamma) \int_{\lambda}^{\gamma} \frac{1}{\mathrm{v}} \mathrm{dv} \rightarrow \infty \text { as } \lambda \rightarrow 0 .
$$

In case $\alpha>0$, use a similar estimation, but with the roles of $\mathrm{W}(\mathrm{v})$ and $\frac{1}{\mathrm{v}}$ interchanged and apply the "asymptotic nonlinearity" hypothesis. Fix $\hat{\alpha} \in(\alpha, \beta)$ and define $\hat{\mathrm{q}}$ and $\mathrm{q}_{\lambda}$, respectively, by $\mathrm{U}^{\prime}(\hat{\mathrm{q}})=\hat{\alpha}$ and $\mathrm{U}^{\prime}\left(\mathrm{q}_{\lambda}\right)=\lambda$. Comparing areas under the graphs of $U^{\prime}$ and its inverse $W$, we see that

$$
\begin{aligned}
\int_{\lambda}^{\beta} \frac{\mathrm{W}(\mathrm{v})}{\mathrm{v}} \mathrm{dv} & \geq \int_{\lambda}^{\hat{\alpha}} \frac{\mathrm{W}(\mathrm{v})}{\mathrm{v}} \mathrm{dv} \geq \frac{1}{\hat{\alpha}} \int_{\lambda}^{\hat{\alpha}} \mathrm{W}(\mathrm{v}) \mathrm{d} \mathbf{v} \geq \frac{1}{\hat{\alpha}} \int_{\hat{\mathrm{q}}}^{\mathrm{q}_{\lambda}}\left(\mathrm{U}^{\prime}(\mathrm{q})-\alpha\right) \mathrm{dq} \\
& =\frac{1}{\hat{\alpha}}\left(\left(\mathrm{U}\left(\mathrm{q}_{\lambda}\right)-\alpha \mathrm{q}_{\lambda}\right)-(\mathrm{U}(\hat{\mathrm{q}})-\alpha \hat{\mathrm{q}})\right) .
\end{aligned}
$$

Assertion $\mathrm{i}$ now follows since, $\mathrm{q}_{\lambda} \rightarrow \infty$ as $\lambda \rightarrow \alpha$ and, by hypothesis, $\lim _{\mathrm{q} \rightarrow \infty} \mathrm{U}(\mathrm{q})-\alpha \mathrm{q}=$ $+\infty$.

Assertion ii. $\lim _{\lambda \rightarrow \beta} \int_{\lambda}^{\beta} \frac{\mathrm{W}(\mathrm{v})}{\mathrm{v}} \mathrm{dv}=0$.

In case $\beta<\infty$, fix $\lambda_{0} \in(\alpha, \beta)$ and observe that for $\lambda>\lambda_{0}$

$$
\int_{\lambda}^{\beta} \frac{\mathrm{W}(\mathrm{v})}{\mathrm{v}} \mathrm{dv} \leq \frac{\mathrm{W}\left(\lambda_{\mathrm{o}}\right)}{\lambda_{\mathrm{o}}} \int_{\lambda}^{\beta} \mathrm{dv} \rightarrow 0 \text { as } \lambda \rightarrow \beta \text {. }
$$


In case $\beta=+\infty$, observe first that $\int_{\lambda_{\mathrm{O}}}^{\infty} \mathrm{W}(\mathrm{v}) \mathrm{dv}<\infty$. To see this, compare the areas
under $\mathrm{U}^{\prime}$ and $\mathrm{W}:$

$$
\int_{\lambda_{0}}^{\infty} \mathrm{W}(\mathrm{v}) \mathrm{dv}=\int_{0}^{\mathrm{q}_{\mathrm{o}}}\left(\mathrm{U}^{\prime}(\mathrm{q})-\lambda_{\mathrm{o}}\right) \mathrm{dq}<\int_{0}^{\mathrm{q}_{\mathrm{o}}} \mathrm{U}^{\prime}(\mathrm{q}) \mathrm{dq}=\mathrm{U}(\mathrm{q})-\mathrm{U}(0)<\infty .
$$

Now for $\lambda>\lambda_{0}$

$$
\int_{\lambda}^{\infty} \frac{W(v)}{v} d v \leq \frac{1}{\lambda} \int_{\lambda}^{\infty} W(v) d v \leq \frac{1}{\lambda} \int_{\lambda_{0}}^{\infty} W(v) d v
$$

So as $\lambda \rightarrow \beta$, the integral on the left approaches zero.

The issue of existence of an optimal solution to the resource extraction problem can now be resolved. Suppose an initial stock size $x_{0}$ and a utility function $U$ satisfying a standard set of assumptions (i) - (iv) are given. Under the additional assumption of asymptotic nonlinearity, an extraction function $q(t)=W\left(\lambda e^{\delta t}\right)$ has been produced which satisfies the necessary conditions of the Maximum Principle. Such a function is unique, but is it optimal? To see that it is, let $B=q(0)$. Theorem 3.1 implies that admissible extraction functions can be limited to those satisfying $q(t) \in[0, B]$ so that assumption A12 of Toman [5] is satisfied. The other conditions of Toman's Theorem 2 are easily verified and therefore an optimal solution exists. This discussion is summarized in the following theorem.

THEOREM 3.2. Assume $U$ satisfies the conditions (i) - (iv). In case $\alpha>0$, assume additionally that $\mathrm{U}$ satisfies $\lim _{\mathrm{q} \rightarrow \infty} \mathrm{U}(\mathrm{q})-\alpha \mathrm{q}=+\infty$. Then there exists an optimal solution to the problem of section 2 .

Finally we relate our results to those in the literature. Lozada's thesis [3], of course, contains much more than existence results for the simple model discussed here. However his result on the problem considered here is basically that if $\alpha=0$ or if $\inf \mathrm{W}(\mathrm{v})>0$ then the resource will be exhausted. So his theorem does not apply (and Theorem 3.1 does) to utility functions such as $\mathrm{U}(\mathrm{q})=\ln (\mathrm{q}+1)+\mathrm{q}, \mathrm{U}(\mathrm{q})=\sqrt{\mathrm{q}}+\mathrm{q}$, or $\mathrm{U}(\mathrm{q})=\tan ^{-1} \mathrm{q}$, while our theorem would not apply to functions such as $U(q)=\sqrt{q^{2}-1}$. Thus our results are distinct from Lozada's.

Epstein's paper [2] is also not principally concerned with existence results for optimal control problems. So some of his conditions are phrased to be more applicable to his topic of analyzing risk aversion. Lemma 1 of [2] is the result of relevance here. The two conditions which assure existence of an optimal solution are

$$
\int_{c_{0}}^{\infty} \frac{-c U^{\prime \prime}(c)}{U^{\prime}(c)} d c=\infty \quad \text { and }
$$

$$
\int_{0}^{c_{1}} \frac{-c U^{\prime \prime}(c)}{U^{\prime}(c)} d c<\infty
$$

By changing variables $\left(x=U^{\prime}(c)\right)$, it follows that these two conditions are essentially the two assertions in the proof of Theorem 3.1. In case $\alpha\left(=\mathrm{U}^{\prime}(\infty)\right)$ is positive, (IC1) is equivalent to the condition that the utility function is asymptotically nonlinear. On the other hand if $\alpha=0$, then Epstein's conditions hold automatically. Clearly the conditions of Theorem 3.1 are more intuitive and geometrical than the integral conditions of Epstein. 
Lastly, Botteron and Dacorogna [6] prove an existence theorem for the problem of minimizing $\int_{0}^{1} g\left(t, v^{\prime}(t)\right) d t \quad$ for functions $v \in C^{1}([0,1])$ satisfying $v(0)=0, \quad v(1)$ $=S$, and $\mathrm{v}^{\prime}(\mathrm{t}) \geq \beta \geq 0$. The hypotheses on $\mathrm{g}$ fall into 2 groups. The first group includes regularity and convexity conditions and are more general than our conditions. (This is due partly to our more specialized economic applications. Our theorems can be generalized to include discounting functions more varied than $\mathrm{e}^{-\delta t}$.) The second hypothesis on $g$ in [6] is a condition on $\partial \mathrm{g}(\mathrm{t}, \mathrm{y}) / \partial \mathrm{y}$. While similar in spirit to our conditions on $\mathrm{U}^{\prime}$ the conditions are quite distinct due to the fact that the problem in [6] has a fixed final state and a fixed final time. In our optimal extraction problem the final time, in particular, is one of the choice variables. (Especially in the case that $\mathrm{T}=+\infty$, the condition in [6] is inapplicable to our problem.) As a result, the conditions of [6] and the current paper are different and in fact are complementary in that taken together, they cover both the free and fixed endpoint problems.

\section{REFERENCES}

1. DASGUPTA, P.S. and Heal, G.M. Economic Theory and Exhaustible Resources, Cambridge University Press, 1979.

2. EPSTEIN, L.G. Decreasing absolute risk aversion and utility indices derived from cakeeating problems, I. Economic Theory 29 (1983) 245-264.

3. LOZADA, G.A. Equilibrium in Exhaustible Resource Industries, Dissertation, Stanford University, 1987.

4. YAARI, M. On the existence of an optimal plan in a continuous-time allocation process, Econometrica $\underline{32}$ (1964) 576-590.

5. TOMAN, M.A. Optimal control with an unbounded horizon, J. Economic Dynamics and Control 2 (1985) 291-316.

6. BOTTERON, B. and DACOROGNA, B. Existence of Solutions for a Variational Problem Associated to Models in Optimal Foraging Theory, J. Math. Anal. Appl. $\underline{147}(1990)$ 263-276. 


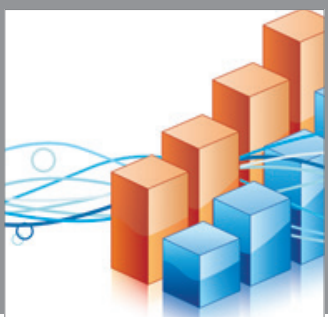

Advances in

Operations Research

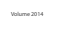

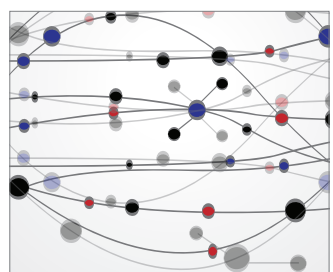

\section{The Scientific} World Journal
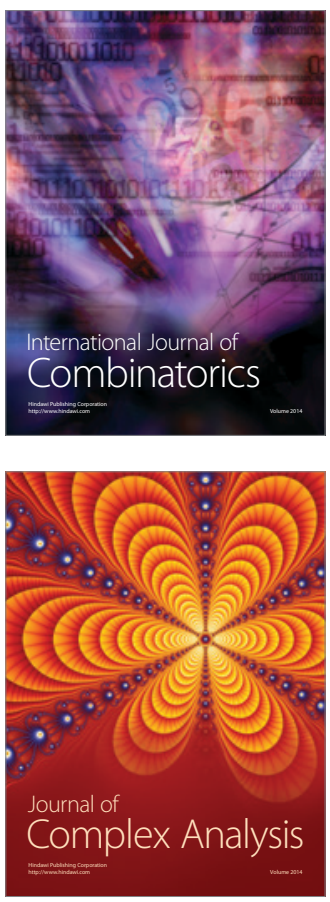

International Journal of

Mathematics and

Mathematical

Sciences
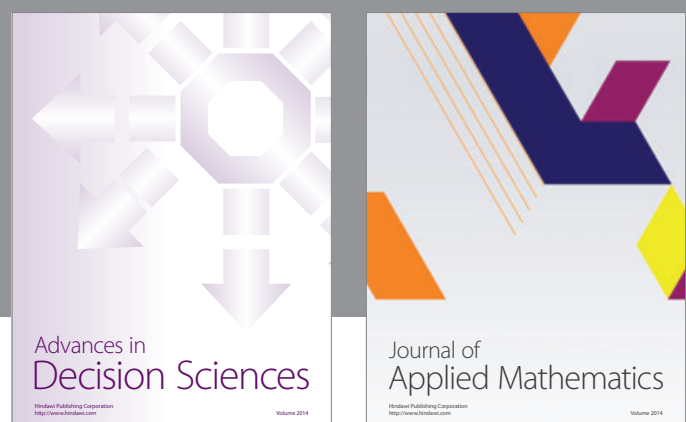

Journal of

Applied Mathematics
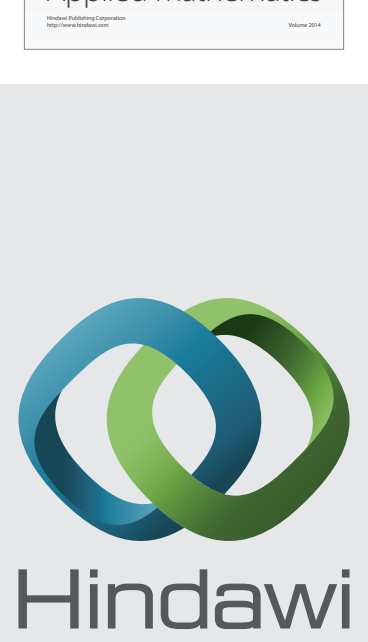

Submit your manuscripts at http://www.hindawi.com
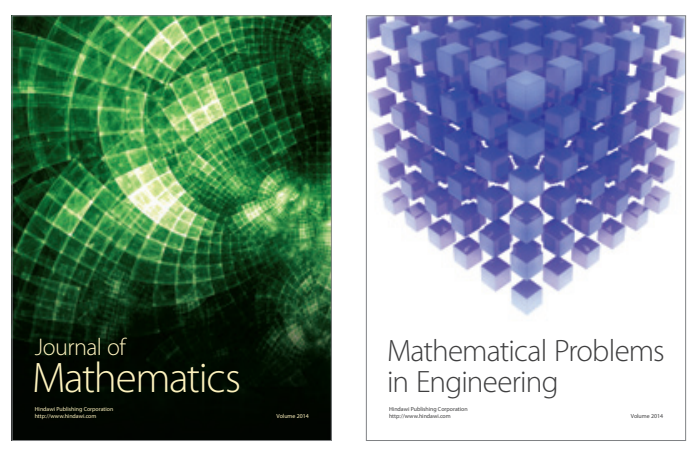

Mathematical Problems in Engineering
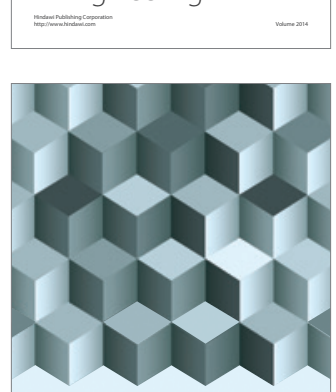

Journal of

Function Spaces
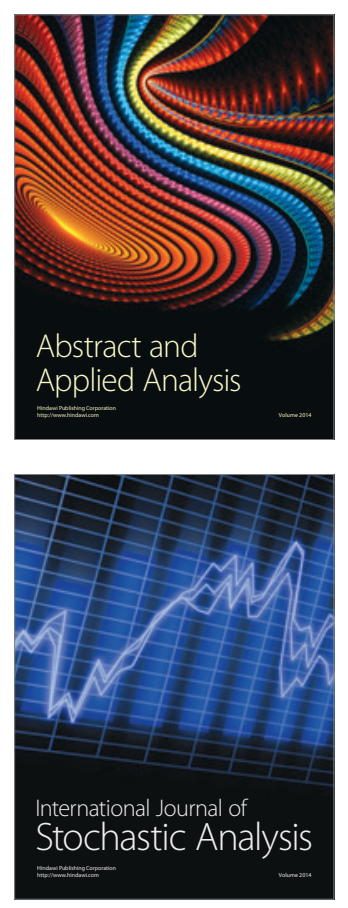

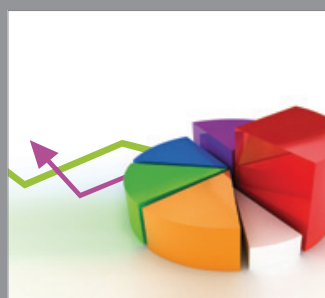

ournal of

Probability and Statistics

Promensencen
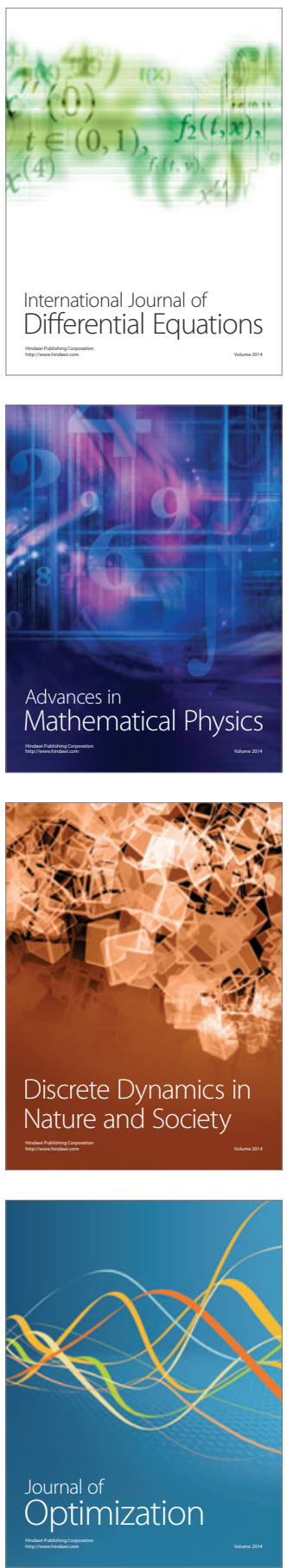